DOI: $10.15503 /$ jecs20162.424.439

\title{
ANALYSIS OF FISCAL COMPETITIVENESS IN LITHUANIA'S MUNICIPALITIES
}

\author{
ROBERTA BAJORŪNIENE்
}

(c) $\odot \otimes \Theta$

Šiauliai University, Social Sciences Faculty, P. Višinskio str. 19, 77156 Šiauliai, Lithuania

E-mail address: bajoruniene.roberta@gmail.com

\section{DALIA RUDYTE்}

Šiauliai University, Social Sciences Faculty, P. Višinskio str. 19, 77156 Šiauliai, Lithuania

E-mail address: daliarudyte@gmail.com

\section{SOLVEIGA SKUNČIKIENĖ}

Šiauliai University, Social Sciences Faculty, P. Višinskio str. 19, 77156 Šiauliai, Lithuania

E-mail address: skusolveiga@gmail.com

\begin{abstract}
Nowadays municipal revenue management and administrative issues are more important and problematic. It is noted that in Lithuania's separate municipalities collected budget revenues, formed budget expenditure, set borrowing limits, allocated from the state budget financial resources (grants) are significantly different. In order to determine reasons for these differences, it is appropriate to analyze the municipal fiscal competitiveness and identify fiscal competitiveness factors of individual Lithuanian municipalities. Understanding municipal fiscal competitiveness factors helps to explain why some municipalities more successfully than others increase tax revenue level and financial - social opportunities of their populations.

This scientific article is funded by the Research Council of Lithuania according to the project "The evaluation of municipalities' fiscal competitiveness in the context of economic growth" (2015-2018), registration No. MIP-013/2015.
\end{abstract}

Key words: Municipality, Fiscal competitiveness, Budget revenue, Expenditure, Surplus value per person.

\section{INTRODUCTION}

In Lithuania municipal distribution of tax revenues is always assessed, but scientific studies are fragmentary involving only a few municipali- 
ties, not enough focus on the causes and effects on the country's economic growth. R. Civinskas, and L. Tolvaišis (2006) estimated the municipal financial autonomy and interbudgetary redistribution of funds, but did not evaluate its impact on municipal fiscal competitiveness. J. Baltušnikienė (2004); G. Davulis (2006) analyzed theoretical and methodological aspects of the fiscal decentralization, but their works were declarative. R. Balvočiūtè, and A. Makauskaite (2012) assessed the regional differentiation of municipalities' finances; however, it lacked a deeper assessment of causes and problems' solutions. A. Žalevičienè (2012) analyzed the regional policies related to foreign investments, J. Baltušnikienè, and A. Astrauskas (2009) emphasized competence of municipal competitiveness, A. Misiūnas, and Z. Svetikas (2003) studied the socio-economic problems with municipalities and they used average index (by comparing to state average) and MIN/MAX index (by comparing municipalities minimum and maximum values) methods. To sum up, in the scientific literature authors usually analyse several municipalities or fiscal decentralization in the context of tax collection. So there is a lack of complex evaluation revealing opportunities of the municipal fiscal competitiveness that are geared to the country's economic growth.

Foreign authors fiscal competitiveness analyzed in Belgium (Gerard, \& Van Malderen, 2012), Czech Republic (Sedmihradska, \& Čabla, 2013), Spain (Delgado, \& Mayor-Fernández, 2009), USA (Hendrick, Wu, \& Jacob, 2007), Canada (Brett, \& Tardif, 2005), Portugal (da Silva Costa, \& Carvalho, 2013), France (Charlot, Paty, \& Piguet, 2010), Finland (Lyytikainen, 2011), Switzerland (Bessard, 2008), Germany (Janeba, \& Osterloh 2012), and other countries (The Kosovo municipal competitiveness index report, 2013). In the scientific literature authors also analyzed all tax revenues (Bessard, 2008) or usual revenues from property tax (Brett, \& Tardif, 2005; Sedmihradska, \& Čabla, 2013, da Silva Costa, \& Carvalho, 2013). A. Monten, and M. Thum (2010); E. Janeba, and S. Osterloh (2012) focused on fiscal competitiveness modeling.

The study of originality reflects the fact that in Lithuania authors usually do not analyze municipal fiscal competitiveness. Created municipal fiscal competitiveness indices are either very common (e.g. municipal competitiveness index created by Lithuanian Free Market Institute) or covering only a separate part of activities. Municipal community index analyzed S. Nefas (2013), other authors (Domarkas, Laukaitytė, \& Mačiukas, 2012) set out the ranking of municipalities by the development of e-government index; A. Misiūnas, and Z. Svetikas (2003) justified the use of integrated index to determine Lithuanian problematic regions. Foreign scientists and institutions (e.g. National Competitiveness Council of the Philippines, U.S. Agency for International Development, European Commission) applied different municipal rating methodologies as well as areas for the evaluation of the municipality. From this originality and relevance of the research we highlight a scientific problem - there is no municipal fiscal competitiveness assessment methodology. Because of this, we decided to reveal problematic issues: how to describe municipal fiscal competitiveness, what factors determine the fiscal 
competitiveness and how Lithuanian municipalities distribute into groups in accordance with the surplus value in production costs and budget revenues and expenditures? That is why the aim of our research is to find out how we can analyze and what is the situation in Lithuanian municipalities' fiscal competitiveness. In the article we used ranking and average index methods (comparing to state average value). These methods will help us to make an analysis and to rank each municipality according to the surplus value and budget revenues and expenditures.

\section{LITERATURE REVIEW}

The scientific literature suggests that the purpose of municipalities is to meet citizens' (as well as business enterprises) societal needs by producing and (or) organizing public goods. And the mission can be defined as within public administration entities to create, develop and reform the economic, social, cultural and ecological controlled territory structure by combining public and private levels of the government (Baltušnikienè, \& Astrauskas, 2009). We notice that in Lithuania's separate municipalities collected budget revenues, formed budget expenditure, set borrowing limits, allocated from the state budget financial resources (grants) are significantly different. In order to determine reasons for these differences, we will appropriately define a conception of municipal fiscal competitiveness.

In the literature there are debates on the definition of the concept of competitiveness (especially, fiscal competitiveness). According to J. Šeputienè, and K. Brazauskienè (2013), competitiveness can be attributed both to the country's well-being (higher income, better quality of life) as well as with local conditions that promote economic growth (cheaper resources, stable labor costs). Also, competitiveness can be analyzed at different levels: company, industry, region or country. J. Bruneckienè (2010) argued that some authors paid more attention to the competitiveness of macro factors such as macroeconomic environment, the level of infrastructure, other authors emphasized business impact on competitiveness or "intangible" factors (tolerance, diversity, creativity level).

We can say that the municipal fiscal (redistributive) function reveals the fact that the budget of income is based on taxes, and received taxpayer funds are redistributed between the municipal citizens, business companies and sphere of activities. A. Novošinskienè (2013) notices that for each municipality it is very important to collect more tax revenues, otherwise it will be difficult to perform its functions. According to the recent version of The Law on the Budget Structure, municipal budgets' accumulated funds are necessary for statutory functions (e.g. education, culture, health, social welfare programs, and others) and for state delegated functions, so because of this each municipality tries to make an independent budget, which is approved without a deficit (The Law on the Budget Structure, 2014). 
I. Hawkesworth, R. Emery, J. Wehner, and J. Saegert (2010) emphasize that Lithuania has separate budgets for the state (central government), municipalities (local government) and social insurance funds. The state budget and main financial indicators of municipal budgets are approved in the Law on Approval of the Financial Indicators of the State Budget and Municipal Budgets. The state budget, as approved by parliament, covers the revenue and expenditures of the government ministries and other budgetary institutions, including state transfers to municipalities. The municipal budget is approved by the municipal council and covers municipal revenues and funds transferred from the state for delegated functions.

The Law on the Methodology of Determination of Municipal Budgetary Revenues establishes the municipal budget as well as determines three local revenue sources: tax revenues, non-tax revenues and grants. Tax and non-tax revenues are allocated to perform municipal independent functions. Another income group is the state budget grant for reducing disparities of income and expenditure between municipalities. Grant rates for municipalities are approved by law. According to this, we notice that the biggest part of the revenue a municipality gets is not from taxes (as it should be), but from grants, so this fact reduces the municipal financial autonomy or as we call "fiscal competitiveness".

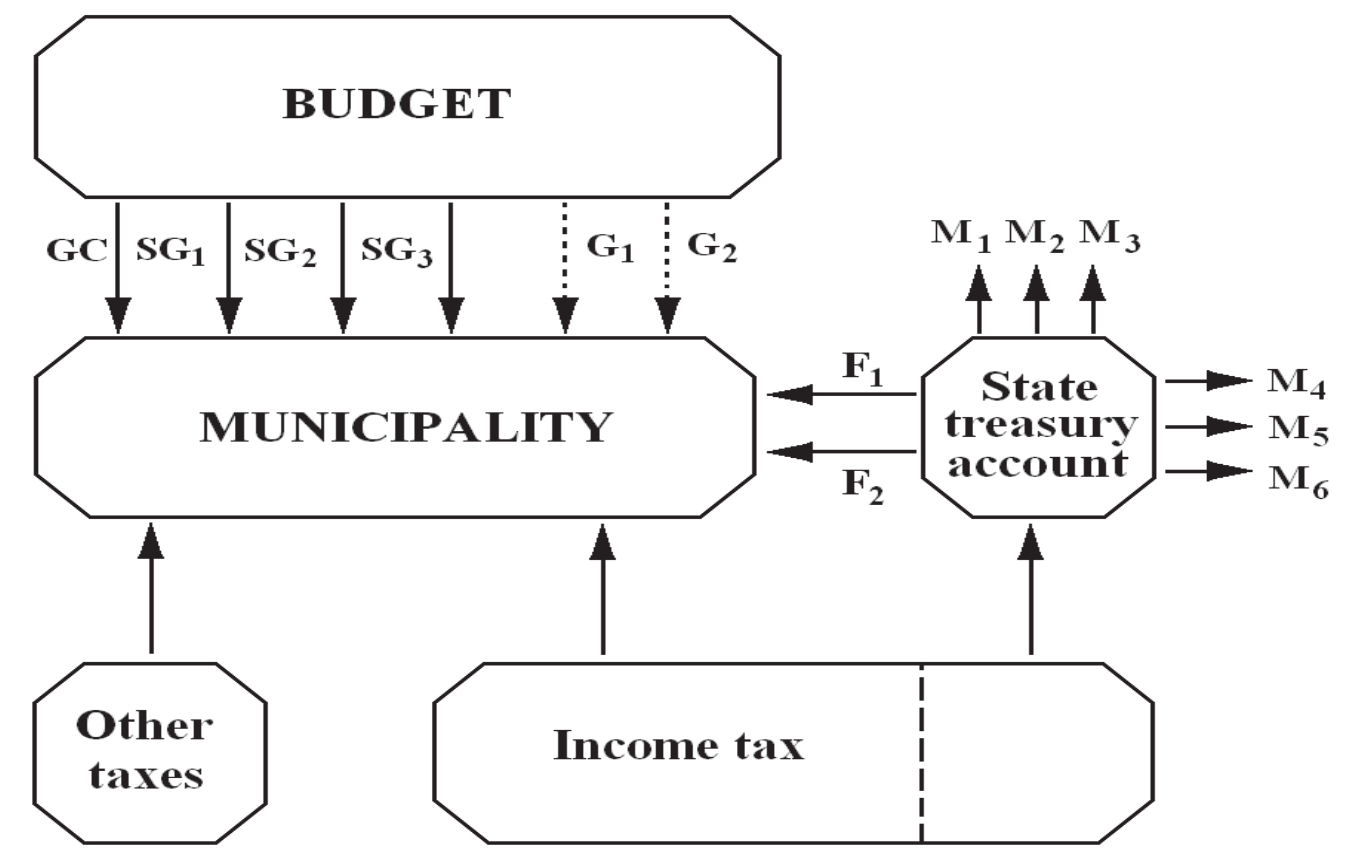

Fig.1. The municipal financing from taxes and grants. Source: Authors created.

where: $M_{1}, M_{2}, \ldots, M_{n}$ - funds from the state treasury account for each municipality $\left(F_{1}+F_{2}\right)$; $\mathrm{F}_{1}$ - funds from the state treasury account to the municipality for equalization of personal income tax; $\mathrm{F}_{2}$ - funds from the state treasury account to the municipality for equalization of differences in cost structures; $\mathrm{SG}_{1}, \mathrm{SG}_{2^{2}} \mathrm{SG}_{3}$ - special purpose grants to the municipality for implementation of public functions $\left(\mathrm{SG}_{1}\right)$, general education $\left(\mathrm{SG}_{2}\right)$, programs $\left(\mathrm{SG}_{3}\right) ; \mathrm{G}_{1}, \mathrm{G}_{2}-$ grants to the municipality if there is lack of $\mathrm{F}_{1}$ or $\mathrm{F}_{2} ; \mathrm{GC}$ - the general grant compensation. 
As we see from Figure 1, the income tax of inhabitants is distributed through the budget, because one of the main Lithuanian Development Goals is to reduce social and economic disparities between regions and within regions, to maintain the identity of these regions, but also to promote the sustainable development of the whole of Lithuania. The Law on the Methodology on Determination of Municipal Budgetary Revenues does not allow municipalities to collect in their budgets more money than it was planned. The first two kinds of income (tax revenue and non-tax revenue) may be called as own income, while the last one - grants - are state designated funds for municipalities to deliver their functions. Unfortunately, the unutilized balance of the latter is to be returned to the state budget at the end of a year (Davulis, 2009). According to R. Ginevičius, N. Slavinskaitė, and D. Gedvilaitè (2014), these revenues enable municipalities to dispose of their budget: they can independently use revenues allocated by the state and acquire some financial resources to ensure the fulfillment of assigned tasks. However, we emphasize that the municipal financial management is very complicated and governed by various laws that restrict the autonomy of local government.

The municipal personal income tax and cost structure alignment does not encourage municipal-donors to increase their income, hampers their natural development. Economically weaker municipalities depend on grants, and this discourages possibility for the improvement of the economic situation. Municipal politicians usually active in preparing the budget, publicly express dissatisfaction with the lack of funds, which municipalities really need, i.e. they seek to attract more funds to municipalities (Civinskas, \& Tolvaišis, 2006). In order to make financial support for economically weaker municipalities and not to hamper the financial autonomy of economically stronger municipalities, we suggest equalizing differences of personal income tax and cost structures not at the expense of other municipal income, but on general grants from the state budget.

After the evaluation of literature review, we could highlight that the municipal fiscal competitiveness could be defined as the ability of the municipality to redistribute budget revenues whereby the residents, business companies and all spheres of activity could be able to pay more taxes to the municipal budget.

\section{ASSESSMENT METHODOLOGY}

To accomplish an analysis we used statistical data from the Lithuanian statistical system and from the Association of local authorities in Lithuania. Lithuanian municipal fiscal competitiveness we analyzed through budget revenues, expenditures and created surplus value in manufacturing prices.

Research sample: in this article we will analyze 60 Lithuanian municipalities. 
Time period: 2005-2014. This choice of investigation period was due to aspiration to reveal Lithuanian municipal financial situation after admission to the European Union (from 2004).

Methods: comparative analysis of statistical data, ranking and average method.

A. Lazauskienè (2008) emphasized, that in the scientific literature authors paid a lot of attention on the problem of the size of municipalities. Larger municipalities can perform more functions; provide more services; larger capacity of realization of various development projects and collection of more tax revenue for the municipal budget. What is more, larger municipalities have cheaper costs of public service delivery, administrative machinery and infrastructure maintenance. Because of this, we decided to apply a ranking method in accordance with municipal size, population, budget revenues (tax, non-tax revenues and received grants) and budget expenditure by functions. J. Krumplytė, and A. Rimkevičienė (2004) noticed, that ranking in accordance with each individual indicator's value estimates municipalities from best place (first rank) to worst (last rank). This method allows for disclosure of certain municipal dimension of fiscal competitiveness.

Scientific literature (Bruneckienè, 2010; Gardiner, Martin, \& Tyler, 2004; Martin, 2003) notices that the competitiveness can be analyzed at different levels: company, industry, region or country, but municipal economic competitiveness and fiscal aspects are not sufficiently studied. Municipalities act freely and independently in their defined territory, establish and approve its budget; the municipal council has the right to the law within the limits and procedures to establish local levies and levies' concessions. The state supports municipalities - there is a grant scheme and a borrowing mechanism, which shows municipal financial autonomy and the opportunity to decide financial issues, but within the borrowing limits. In order to reveal the municipality's economic competitiveness we also adapted the ranking method based on the surplus value in manufacturing prices per capita. Surplus value indicator was chosen due to the fact that in the literature authors usually relate competitiveness to productivity, which reflects the surplus value per capita.

We used the average method because our survey sample was relatively high (60 municipalities) and analysis period was long (2005-2014). The average method showed what was the Lithuanian municipality's rank, compared with the average rate of all Lithuanian municipalities. A. Misiunas, and Z. Svetikas (2003) showed the formula of average method:

$$
I_{i j}(t)=\frac{x_{j}(t)}{x_{\mathbb{E}, j}(t)}
$$

where: $x_{i j}(t)$ - the municipality's value of the index for a period of time $(t)$; $x_{L T, j}(t)$ - the country's average value of the index during the analysis period $(\mathrm{t})$. 
In summary, the assessment methodology of municipal fiscal competitiveness emphasizes that there are many indicators which show the economic and financial situation of municipalities, but the most important is the surplus value and budget indicators (income and expenditure). According to this, we will analyze Lithuanian municipal fiscal competitiveness.

\section{ANALYSIS OF FISCAL COMPETITIVENESS IN LITHUANIA}

In the Republic of Lithuania administrative units are counties and municipalities. At present, the Republic of Lithuania has 10 counties and 60 municipalities. Distribution of municipalities by territory and population is shown in Table 1 and Table 2. According to A. Lazauskienè (2008), an administrative unit size is almost always defined by two parameters: size of territory and population.

We ranked into five groups Lithuanian municipalities according to the size of territory. The first group had the three largest municipalities in Lithuania: Varèna district, Panevėžys district and Vilnius district. The second group consisted of 10 municipalities (rank from 4 to 13). The third group was the largest and consisted of 25 municipalities (rank from 14 to 38), the fourth group consisted of 10 municipalities (rank was from 39 to 48) and the last one - fifth group - consisted of 12 municipalities (rank was from 49 to 60). All Lithuania territory is 65,3 thousand sq. km. Ranking was performed of every $500 \mathrm{sq}$. km. Small municipalities have a flexible administrative apparatus, and public affairs are less bureaucratic. In addition, the smaller territorial unit strengthens the competition between areas and promotes local government dynamism and development of new ideas.

According to average population, we ranked Lithuanian municipalities into eight groups (Table 2). Ranking was performed of every 10000 inhabitants. The first group consists of 7 the most important Lithuanian municipalities. The largest group is sixth and it consists of 17 municipalities. The smallest group is the last one (only four municipalities rank from 57 to 60).

Lithuanian major cities' municipalities are largest according to population. The biggest part of the local budget tax revenue consists of revenue from personal income tax, so population of municipalities is an important indicator. Larger population means greater tax revenue received by the municipality.

Based on scientific studies, which analyze development and trends of fiscal policy and taxation, it can be emphasized that both the state and municipal financial efficiency is important for economic productivity. It is important to shape the financing of the budget - the tax structure - to ensure the collection of tax revenues and can be beneficial for competitiveness growth (Stankevičius, 2014). 
Table 1. Lithuanian municipalities' ranking by the size of the territory. Source: Own research.

\begin{tabular}{|c|c|c|c|c|c|c|c|c|c|c|c|c|c|c|}
\hline Municipality & $\begin{array}{c}\text { Territory } \\
(\mathbf{2 0 1 4 )}, \\
\mathrm{km} 2\end{array}$ & Rank & Municipality & $\begin{array}{c}\text { Territory } \\
(2014), \\
\text { km2 }\end{array}$ & Rank & Municipality & $\begin{array}{c}\text { Territory } \\
(2014), \\
\text { km2 }\end{array}$ & Rank & Municipality & $\begin{array}{c}\text { Territory } \\
(2014), \\
\text { km2 }\end{array}$ & Rank & Municipality & $\begin{array}{c}\text { Territory } \\
(\mathbf{2 0 1 4}), \\
\mathrm{km} 2\end{array}$ & Rank \\
\hline $\begin{array}{l}\text { Varêna } \\
\text { district }\end{array}$ & 2218 & 1 & $\begin{array}{c}\text { Jurbarkas } \\
\text { district }\end{array}$ & 1507 & 13 & $\begin{array}{l}\text { Pakruoj15 } \\
\text { district }\end{array}$ & 1316 & 25 & $\begin{array}{c}\text { Kupiskis } \\
\text { district }\end{array}$ & 1080 & 37 & Druskinmkai & 454 & 49 \\
\hline $\begin{array}{c}\text { Panevežys } \\
\text { district }\end{array}$ & 2179 & 2 & $\begin{array}{l}\text { Kaunas } \\
\text { district }\end{array}$ & 1496 & 14 & $\begin{array}{l}\text { Lazdijai } \\
\text { district }\end{array}$ & 1309 & 26 & $\begin{array}{l}\text { Prienai } \\
\text { district }\end{array}$ & 1031 & 38 & Kalvarija & 441 & 50 \\
\hline $\begin{array}{l}\text { Vilnius } \\
\text { district }\end{array}$ & 2129 & 3 & $\begin{array}{l}\text { Salcminkai } \\
\text { district }\end{array}$ & 1491 & 15 & $\begin{array}{l}\text { Pasvalys } \\
\text { district }\end{array}$ & 1289 & 27 & $\begin{array}{l}\text { Kretinga } \\
\text { district }\end{array}$ & 989 & 39 & Vilnius city & 401 & 51 \\
\hline $\begin{array}{l}\text { Rokisikis } \\
\text { district }\end{array}$ & 1807 & $4-5$ & $\begin{array}{l}\text { Biržai } \\
\text { district }\end{array}$ & 1476 & 16 & $\begin{array}{l}\text { Vilkaviškis } \\
\text { district }\end{array}$ & 1259 & 28 & $\begin{array}{l}\text { Jonzva } \\
\text { district }\end{array}$ & 944 & 40 & Kaunas city & 157 & 52 \\
\hline $\begin{array}{l}\text { Siauliai } \\
\text { district }\end{array}$ & 1807 & $4-5$ & $\begin{array}{l}\text { Sakiai } \\
\text { district }\end{array}$ & 1453 & 17 & $\begin{array}{l}\text { Utena } \\
\text { district }\end{array}$ & 1230 & 29 & $\begin{array}{l}\text { Shruodas } \\
\text { district }\end{array}$ & 911 & 41 & Birśtonas & 124 & 53 \\
\hline $\begin{array}{l}\text { Anykšciai } \\
\text { district }\end{array}$ & 1765 & 6 & $\begin{array}{l}\text { Ignalina } \\
\text { district }\end{array}$ & 1447 & 18 & $\begin{array}{l}\text { Mažeikiai } \\
\text { district }\end{array}$ & 1220 & 30 & $\begin{array}{l}\text { Sirvmtos } \\
\text { district }\end{array}$ & 906 & 42 & Klapeda city & 98 & 54 \\
\hline $\begin{array}{l}\text { Siluté } \\
\text { district }\end{array}$ & 1706 & 7 & $\begin{array}{l}\text { Telsiai } \\
\text { district }\end{array}$ & 1439 & 19 & $\begin{array}{l}\text { Trakai } \\
\text { district }\end{array}$ & 1208 & 31 & $\begin{array}{l}\text { Alomene } \\
\text { district }\end{array}$ & 844 & 43 & Neringa & 90 & 55 \\
\hline $\begin{array}{l}\text { Kelmé } \\
\text { district }\end{array}$ & 1705 & 8 & $\begin{array}{l}\text { Alytus } \\
\text { district }\end{array}$ & 1404 & 20 & $\begin{array}{l}\text { Silale } \\
\text { district }\end{array}$ & 1188 & 32 & Marijampole & 755 & 44 & Siauliai city & 81 & 56 \\
\hline $\begin{array}{l}\text { Svencionys } \\
\text { district }\end{array}$ & 1692 & 9 & $\begin{array}{l}\text { Ulmerge } \\
\text { district }\end{array}$ & 1395 & 21 & $\begin{array}{l}\text { Taurage } \\
\text { district }\end{array}$ & 1179 & 33 & Rietavas & 586 & 45 & Palanga city & 79 & 57 \\
\hline $\begin{array}{c}\text { Kedainiai } \\
\text { district }\end{array}$ & 1677 & 10 & $\begin{array}{l}\text { Moletai } \\
\text { district }\end{array}$ & 1367 & 22 & $\begin{array}{l}\text { Jontskisis } \\
\text { district }\end{array}$ & 1152 & 34 & Kazlụ Rũda & 555 & 46 & Yisagmas & 58 & 58 \\
\hline $\begin{array}{l}\text { Radviliskis } \\
\text { district }\end{array}$ & 1635 & 11 & $\begin{array}{l}\text { Klaipeda } \\
\text { district }\end{array}$ & 1336 & 23 & $\begin{array}{l}\text { Plunge } \\
\text { district }\end{array}$ & 1105 & 35 & Pagegtai & 537 & 47 & Panevezys city & 50 & 59 \\
\hline $\begin{array}{c}\text { Rasemiai } \\
\text { district }\end{array}$ & 1573 & 12 & $\begin{array}{l}\text { Zarasai } \\
\text { district }\end{array}$ & 1334 & 24 & $\begin{array}{l}\text { Katśiadorys } \\
\text { district }\end{array}$ & 1087 & 36 & Elektrenai & 509 & 48 & Alytus city & 40 & 60 \\
\hline
\end{tabular}


Table 2. Lithuanian municipalities' ranking by the population (2005-2014). Source: Own research.

\begin{tabular}{|c|c|c|c|c|c|c|c|c|c|c|c|c|c|c|}
\hline Municipality & $\begin{array}{l}\text { Average } \\
\text { population }\end{array}$ & Rank & Municipality & $\begin{array}{c}\text { Average } \\
\text { population }\end{array}$ & Rank & Municipality & $\begin{array}{c}\text { Average } \\
\text { population }\end{array}$ & Rank & Municipality & $\begin{array}{c}\text { Average } \\
\text { population }\end{array}$ & Rank & Municipality & $\begin{array}{c}\text { Average } \\
\text { population }\end{array}$ & Rank \\
\hline Vilnius city & 540298 & 1 & $\begin{array}{l}\text { Telsiai } \\
\text { district }\end{array}$ & 48628 & 13 & $\begin{array}{l}\text { Rasemiai } \\
\text { district }\end{array}$ & 38605 & 25 & Alytus district & 28974 & 37 & $\begin{array}{l}\text { Skuodas } \\
\text { district }\end{array}$ & 21238 & 49 \\
\hline Kaunas city & 326506 & 2 & $\begin{array}{l}\text { Jonava } \\
\text { district }\end{array}$ & 47530 & 14 & $\begin{array}{l}\text { Rokkiskts } \\
\text { district }\end{array}$ & 35928 & 26 & $\begin{array}{c}\text { Svencionys } \\
\text { district }\end{array}$ & 28544 & 38 & $\begin{array}{l}\text { Kupiškis } \\
\text { district }\end{array}$ & 20986 & 50 \\
\hline Klaipeda city & 167406 & 3 & Silute district & 46840 & 15 & $\begin{array}{l}\text { Salcinmkai } \\
\text { district }\end{array}$ & 35183 & 27 & Silale district & 27326 & 39 & $\begin{array}{l}\text { Zarasai } \\
\text { district }\end{array}$ & 19021 & 51 \\
\hline Sizuliai city & 113552 & 4 & $\begin{array}{l}\text { Siauliai } \\
\text { district }\end{array}$ & 45979 & 16 & $\begin{array}{l}\text { Trakai } \\
\text { district }\end{array}$ & 34937 & 28 & Joniškis district & 27116 & 40 & $\begin{array}{l}\text { Ignalina } \\
\text { district }\end{array}$ & 18982 & 52 \\
\hline $\begin{array}{c}\text { Panevezys } \\
\text { city }\end{array}$ & 102864 & 5 & $\begin{array}{l}\text { Taurage } \\
\text { district }\end{array}$ & 45429 & 17 & $\begin{array}{l}\text { Kaisiadoriai } \\
\text { district }\end{array}$ & 34464 & 29 & Varena district & 26298 & 41 & $\begin{array}{l}\text { Sirvintos } \\
\text { district }\end{array}$ & 17994 & 53 \\
\hline $\begin{array}{l}\text { Vilnius } \\
\text { district }\end{array}$ & 94859 & 6 & Utena district & 44331 & 18 & $\begin{array}{l}\text { Kelmé } \\
\text { district }\end{array}$ & 33778 & 30 & Elektrènai & 25611 & 42 & Palanga city & 16030 & 54 \\
\hline $\begin{array}{l}\text { Kaunas } \\
\text { district }\end{array}$ & 85450 & 7 & $\begin{array}{l}\text { Radviliskis } \\
\text { district }\end{array}$ & 44155 & 19 & $\begin{array}{l}\text { Salktat } \\
\text { district }\end{array}$ & 33443 & 31 & $\begin{array}{l}\text { Palkruojis } \\
\text { district }\end{array}$ & 24627 & 43 & Kazlụ Rüda & 13558 & 55 \\
\hline Marijampole & 62903 & 8 & $\begin{array}{l}\text { Villkaviskis } \\
\text { district }\end{array}$ & 43762 & 20 & $\begin{array}{c}\text { Jurbarkas } \\
\text { district }\end{array}$ & 31455 & 32 & Almene district & 24470 & 44 & Kalvarija & 12351 & 56 \\
\hline Alytus city & 62313 & 9 & $\begin{array}{l}\text { Kretinga } \\
\text { district }\end{array}$ & 42408 & 21 & $\begin{array}{l}\text { Prienai } \\
\text { district }\end{array}$ & 30760 & 33 & Visaginas & 23645 & 45 & Pagegiai & 9948 & 57 \\
\hline $\begin{array}{l}\text { Mazeikiai } \\
\text { district }\end{array}$ & 59910 & 10 & $\begin{array}{l}\text { Ukmerge } \\
\text { district }\end{array}$ & 41500 & 22 & $\begin{array}{l}\text { Anykściai } \\
\text { district }\end{array}$ & 29573 & 34 & Lazdijai district & 23061 & 46 & Rietavas & 9017 & 58 \\
\hline $\begin{array}{c}\text { Kedamiai } \\
\text { district }\end{array}$ & 55752 & 11 & $\begin{array}{l}\text { Panevezys } \\
\text { district }\end{array}$ & 39874 & 23 & Biržai district & 29406 & 35 & Druskiminkai & 22416 & 47 & Birštonas & 4688 & 59 \\
\hline $\begin{array}{l}\text { Klaipeds } \\
\text { district }\end{array}$ & 50868 & 12 & $\begin{array}{l}\text { Plunge } \\
\text { district }\end{array}$ & 39160 & 24 & $\begin{array}{l}\text { Pasvalys } \\
\text { district }\end{array}$ & 29283 & 36 & Moletai district & 21329 & 48 & Neringa & 2489 & 60 \\
\hline
\end{tabular}


Table 3. Lithuanian municipalities' ranking by the surplus value per capita (2005-2014). Source: Own research.

\begin{tabular}{|c|c|c|c|c|c|c|c|c|c|c|c|c|c|c|}
\hline Municipality & $\begin{array}{l}\text { Average } \\
\text { surplus } \\
\text { value per } \\
\text { capita, } \\
\text { thousand } \\
\text { EUR }\end{array}$ & Rank & Municipality & $\begin{array}{l}\text { Average } \\
\text { surplus } \\
\text { value per } \\
\text { capita, } \\
\text { thousand } \\
\text { EUR }\end{array}$ & Rank & Municipality & $\begin{array}{l}\text { Average } \\
\text { surplus } \\
\text { value per } \\
\text { capita, } \\
\text { thousand } \\
\text { EUR }\end{array}$ & Rank & Municipality & $\begin{array}{l}\text { Average } \\
\text { surplus } \\
\text { value per } \\
\text { capita, } \\
\text { thousand } \\
\text { ELR }\end{array}$ & Rank & Municipality & $\begin{array}{l}\text { Average } \\
\text { surplus } \\
\text { value per } \\
\text { capita, } \\
\text { thousand } \\
\text { EUR }\end{array}$ & Rank \\
\hline $\begin{array}{c}\text { Vilnius } \\
\text { city }\end{array}$ & 7,658 & 1 & $\begin{array}{c}\text { Kedainiai } \\
\text { district }\end{array}$ & 3,229 & 12 & $\begin{array}{l}\text { Ukmerge } \\
\text { district }\end{array}$ & 2,055 & 24 & $\begin{array}{l}\text { Kupiškis } \\
\text { district }\end{array}$ & 1,367 & 36 & $\begin{array}{l}\text { Anykšciai } \\
\text { district }\end{array}$ & 1,068 & 48 \\
\hline $\begin{array}{c}\text { Klaipeda } \\
\text { city }\end{array}$ & 6,026 & 2 & $\begin{array}{l}\text { Palanga } \\
\text { city }\end{array}$ & 3,207 & 13 & $\begin{array}{l}\text { Trakai } \\
\text { district }\end{array}$ & 2,031 & 25 & $\begin{array}{l}\text { Siauliai } \\
\text { district }\end{array}$ & 1,342 & 37 & $\begin{array}{l}\text { Sakiai } \\
\text { district }\end{array}$ & 1,061 & 49 \\
\hline $\begin{array}{c}\text { Mažeikiai } \\
\text { district }\end{array}$ & 4,908 & 3 & $\begin{array}{l}\text { Utena } \\
\text { district }\end{array}$ & 3,176 & 14 & $\begin{array}{l}\text { Rokiskis } \\
\text { district }\end{array}$ & 1,952 & 26 & $\begin{array}{l}\text { Birżai } \\
\text { district }\end{array}$ & 1,341 & 38 & $\begin{array}{l}\text { Vilkaviskis } \\
\text { district }\end{array}$ & 0,973 & 50 \\
\hline $\begin{array}{c}\text { Kaunas } \\
\text { city }\end{array}$ & 4,795 & 4 & Alytus city & 3,075 & 15 & $\begin{array}{c}\text { Taurage } \\
\text { district }\end{array}$ & 1,810 & 27 & $\begin{array}{l}\text { Molétai } \\
\text { district }\end{array}$ & 1,325 & 39 & $\begin{array}{l}\text { Sirvintos } \\
\text { district }\end{array}$ & 0,955 & 51 \\
\hline Neringa & 4,174 & 5 & Kazlu Ruda & 2,759 & 16 & Rietavas & 1,784 & 28 & Birštonas & 1,287 & 40 & Pagégiai & 0,946 & $\begin{array}{l}52- \\
53\end{array}$ \\
\hline $\begin{array}{l}\text { Paneveżys } \\
\text { city }\end{array}$ & 4,083 & 6 & Marijampole & 2,665 & 17 & $\begin{array}{c}\text { Kaišiadorys } \\
\text { district }\end{array}$ & 1,633 & 29 & $\begin{array}{l}\text { Varena } \\
\text { district }\end{array}$ & 1,280 & 41 & $\begin{array}{l}\text { Alytus } \\
\text { district }\end{array}$ & 0,946 & $\begin{array}{l}52- \\
53 \\
\end{array}$ \\
\hline $\begin{array}{l}\text { Siauliai } \\
\text { city }\end{array}$ & 4,011 & $7-8$ & Druskininkai & 2,504 & 18 & $\begin{array}{l}\text { Kretinga } \\
\text { district }\end{array}$ & 1,623 & 30 & $\begin{array}{c}\text { Paneveżys } \\
\text { district }\end{array}$ & 1,276 & 42 & $\begin{array}{c}\text { Ignalina } \\
\text { district }\end{array}$ & 0,911 & 54 \\
\hline $\begin{array}{l}\text { Klaipeda } \\
\text { district }\end{array}$ & 4,011 & $7-8$ & $\begin{array}{l}\text { Kaunas } \\
\text { district }\end{array}$ & 2,267 & 19 & $\begin{array}{l}\text { Silute } \\
\text { district }\end{array}$ & 1,619 & 31 & $\begin{array}{c}\text { Joniskkis } \\
\text { district } \\
\end{array}$ & 1,231 & 43 & $\begin{array}{l}\text { Kelme } \\
\text { district }\end{array}$ & 0,887 & 55 \\
\hline Visaginas & 3,727 & 9 & $\begin{array}{l}\text { Vilnius } \\
\text { district }\end{array}$ & 2,201 & 20 & $\begin{array}{c}\text { Radviliskis } \\
\text { district }\end{array}$ & 1,597 & 32 & $\begin{array}{l}\text { Prienai } \\
\text { district }\end{array}$ & 1,188 & 44 & $\begin{array}{c}\text { Zarasai } \\
\text { district }\end{array}$ & 0,865 & 56 \\
\hline LITHLANIA & 3,638 & & $\begin{array}{l}\text { Plunge } \\
\text { district }\end{array}$ & 2,154 & 21 & $\begin{array}{c}\text { Raseiniai } \\
\text { district }\end{array}$ & 1,594 & 33 & $\begin{array}{l}\text { Jurbarkas } \\
\text { district }\end{array}$ & 1,142 & 45 & Kalvanja & 0,779 & 57 \\
\hline Elektrenai & 3,551 & 10 & $\begin{array}{l}\text { Akmene } \\
\text { district }\end{array}$ & 2,132 & 22 & $\begin{array}{c}\text { Pakruojis } \\
\text { district }\end{array}$ & 1,478 & $\begin{array}{l}34 . \\
35\end{array}$ & $\begin{array}{c}\text { Pasvalys } \\
\text { district }\end{array}$ & 1,119 & 46 & $\begin{array}{c}\text { Lazdijai } \\
\text { district }\end{array}$ & 0,773 & 58 \\
\hline $\begin{array}{l}\text { Jonava } \\
\text { district }\end{array}$ & 3,450 & 11 & $\begin{array}{l}\text { Telšiai } \\
\text { district }\end{array}$ & 2,116 & 23 & $\begin{array}{l}\text { Svencionys } \\
\text { district }\end{array}$ & 1,478 & $\begin{array}{l}34 . \\
35 \\
\end{array}$ & $\begin{array}{l}\text { Silalè } \\
\text { district }\end{array}$ & 1,085 & 47 & $\begin{array}{l}\text { Skuodas } \\
\text { district }\end{array}$ & 0,748 & 59. \\
\hline & & & & & & & & & & & & $\begin{array}{l}\text { Salčminkai } \\
\text { district }\end{array}$ & 0,743 & 60 \\
\hline
\end{tabular}


Table 4. Lithuanian municipalities' ranking by the budget income per capita (2005-2014). Source: Own research.

\begin{tabular}{|c|c|c|c|c|c|c|c|c|c|c|c|c|c|c|}
\hline Municipality & $\begin{array}{c}\text { Average } \\
\text { budget } \\
\text { revenue } \\
\text { per } \\
\text { capita, } \\
\text { thousand } \\
\text { EUR }\end{array}$ & Rank & Municipality & $\begin{array}{l}\text { Average } \\
\text { budget } \\
\text { revenue } \\
\text { per } \\
\text { capita, } \\
\text { thousand } \\
\text { EUR }\end{array}$ & Rank & Municipality & $\begin{array}{c}\text { Average } \\
\text { budget } \\
\text { revenue } \\
\text { per } \\
\text { capita, } \\
\text { thousand } \\
\text { EUR }\end{array}$ & Rank & Municipality & $\begin{array}{l}\text { Average } \\
\text { budget } \\
\text { revenue } \\
\text { per } \\
\text { capita, } \\
\text { thousand } \\
\text { EUR }\end{array}$ & Rank & Municipality & $\begin{array}{l}\text { Average } \\
\text { budget } \\
\text { revenue } \\
\text { per } \\
\text { capita, } \\
\text { thousand } \\
\text { EUR }\end{array}$ & Rank \\
\hline Neringa & 2,870 & 1 & $\begin{array}{l}\text { Pasvalys } \\
\text { district }\end{array}$ & 0,677 & 12 & $\begin{array}{l}\text { Sakiai } \\
\text { district }\end{array}$ & 0,645 & $23-24$ & $\begin{array}{l}\text { Rokiskis } \\
\text { district }\end{array}$ & 0,623 & 36 & Kaunas city & 0,597 & $48-49$ \\
\hline Birštonas & 1,365 & 2 & $\begin{array}{l}\text { Pakruojis } \\
\text { district }\end{array}$ & 0,676 & $13-14$ & $\begin{array}{l}\text { Telsiai } \\
\text { district }\end{array}$ & 0,643 & 25 & $\begin{array}{l}\text { Ukmerge } \\
\text { district }\end{array}$ & 0,621 & 37 & Kazlu Ruda & 0,597 & $48-49$ \\
\hline Palanga city & 1,017 & 3 & $\begin{array}{l}\text { Varena } \\
\text { district }\end{array}$ & 0.676 & $13-14$ & $\begin{array}{l}\text { Jonava } \\
\text { district }\end{array}$ & 0,641 & 26 & $\begin{array}{c}\text { Vilkaviskis } \\
\text { district }\end{array}$ & 0,619 & 38 & $\begin{array}{l}\text { Paneveżys } \\
\text { district }\end{array}$ & 0,580 & 50 \\
\hline $\begin{array}{l}\text { Akmene } \\
\text { district }\end{array}$ & 0,770 & 4 & $\begin{array}{l}\text { Kelme } \\
\text { district }\end{array}$ & 0,675 & 15 & $\begin{array}{l}\text { Kedainiai } \\
\text { district }\end{array}$ & 0,639 & 27 & Siauliai city & 0,616 & 39 & $\begin{array}{l}\text { Alytus } \\
\text { district }\end{array}$ & 0,577 & 51 \\
\hline Pagégiai & 0,753 & 5 & $\begin{array}{l}\text { Trakai } \\
\text { district }\end{array}$ & 0,674 & 16 & Elektrenai & 0,638 & 28 & $\begin{array}{l}\text { Kaišia dorys } \\
\text { district }\end{array}$ & 0,615 & 40 & $\begin{array}{l}\text { Siauliai } \\
\text { district }\end{array}$ & 0,574 & 52 \\
\hline Druskininkai & 0,724 & 6 & $\begin{array}{l}\text { Skuodas } \\
\text { district }\end{array}$ & 0,665 & 17 & $\begin{array}{l}\text { Sirvintos } \\
\text { district }\end{array}$ & 0,637 & 29 & $\begin{array}{l}\text { Prienai } \\
\text { district }\end{array}$ & 0,614 & 41 & Marijampole & 0,573 & 53 \\
\hline $\begin{array}{l}\text { Zarasai } \\
\text { district }\end{array}$ & 0,700 & 7 & Visaginas & 0,664 & 18 & $\begin{array}{l}\text { Moletai } \\
\text { district }\end{array}$ & 0,635 & 30 & $\begin{array}{l}\text { Plunge } \\
\text { district }\end{array}$ & 0,612 & 42 & $\begin{array}{l}\text { Kretinga } \\
\text { district }\end{array}$ & 0,571 & 54 \\
\hline $\begin{array}{l}\text { Jonikkis } \\
\text { district }\end{array}$ & 0,696 & 8 & $\begin{array}{l}\text { Anykščiai } \\
\text { district }\end{array}$ & 0,661 & 19 & $\begin{array}{l}\text { Mažeikiai } \\
\text { district }\end{array}$ & 0,633 & $31-32$ & Utena district & 0,609 & $43-44$ & $\begin{array}{l}\text { Panevezżys } \\
\text { city }\end{array}$ & 0,558 & 55 \\
\hline $\begin{array}{l}\text { Kupiskkis } \\
\text { district }\end{array}$ & 0,690 & $9-10$ & Rietavas & 0,660 & 20 & Alytus city & 0,633 & $31-32$ & $\begin{array}{l}\text { Taurage } \\
\text { district }\end{array}$ & 0,609 & $43-44$ & $\begin{array}{l}\text { Radviliskis } \\
\text { district }\end{array}$ & 0,553 & 56 \\
\hline $\begin{array}{c}\text { Ignalina } \\
\text { district }\end{array}$ & 0,690 & $9-10$ & $\begin{array}{l}\text { Silutè } \\
\text { district }\end{array}$ & 0,651 & 21 & Biržai district & 0,631 & 33 & Klaipeda city & 0,603 & 45 & $\begin{array}{l}\text { Klaipeda } \\
\text { district }\end{array}$ & 0,529 & 57 \\
\hline $\begin{array}{l}\text { Silale } \\
\text { district }\end{array}$ & 0,687 & 11 & $\begin{array}{l}\text { Lazdijai } \\
\text { district }\end{array}$ & 0,649 & 22 & $\begin{array}{c}\text { Raseiniai } \\
\text { district }\end{array}$ & 0,630 & 34 & $\begin{array}{c}\text { Salcininkai } \\
\text { district }\end{array}$ & 0,602 & 46 & Vilnius city & 0,521 & 58 \\
\hline LITHUANIA & 0,685 & & $\begin{array}{l}\text { Jurbarkas } \\
\text { district }\end{array}$ & 0,645 & $23-24$ & Kalvarija & 0,627 & 35 & $\begin{array}{l}\text { Svencionys } \\
\text { district }\end{array}$ & 0,600 & 47 & $\begin{array}{l}\text { Vilnius } \\
\text { district }\end{array}$ & 0,505 & 59 \\
\hline & & & & & & & & & & & & $\begin{array}{l}\text { Kaunas } \\
\text { district }\end{array}$ & 0,484 & 60 \\
\hline
\end{tabular}


To compare Lithuanian municipalities in terms of productivity (the average surplus value per capita), we grouped all municipalities into 6 groups (ranked on every 1000 euro per capita):

- High productivity (with exceptions) (rank from 1 to 2 );

- High productivity (rank from 3 to 8 );

- The higher-than-average productivity (rank 9 to 15);

- The average productivity (rank from 16 to 25 );

- Lower-than-average productivity (rank from 26 to 49 );

- Low productivity (rank 50 to 60).

High productivity (with exceptions) has two municipalities: the city of Vilnius and Klaipeda city. These municipalities are called "exceptions", because the surplus value is the highest in comparison with other municipalities. Vilnius is the capital of Lithuania, with a population of more than 0,5 million inhabitants. Klaipeda is a seaport city, which is very important for transportation.

In the next stage of our investigation we ranked municipalities according to budget revenues. Budget revenues consist of tax and non-tax revenues, and grants. Municipal budget revenue analysis will help to clarify what is the local government's financial autonomy in Lithuania. We analyzed Lithuanian municipal average budget income per capita (Table 4).

All municipalities are grouped into five groups from the largest budget revenue per capita to the smallest. The first group consists of 3 municipalities (rank from 1 to 3 ), the second group consists of 8 municipalities (rank from 4 to 11), and the third group includes 11 municipalities (rank from 12 to 22). The fourth group is the largest group of municipalities - it consists of 27 municipalities (rank from 23 to 49). And the last group consists of 11 municipalities (rank from 50 to 60). We can mention that only first and second groups exceed the average rate of all Lithuanian municipalities (685 euro).

The highest average budget revenue per capita is in these municipalities: Neringa, Birštonas and Palanga city. It became like this, because of high budget revenues (in absolute value) and the low population. These municipalities are identified as centers of attraction for tourists, because these municipalities are Lithuanian health/holiday resort cities. According to average surplus value per capita, Vilnius and Klaipeda cities created the highest average surplus value per capita, but according to budget revenue these municipalities took a rank of 58 and 45).

In terms of tax revenue, it can be said that the largest tax revenues are received by Neringa, Palanga and Birštonas municipalities. During the analysis period (2005-2014), average tax revenue per capita was 2119 euro, 582 euro and 512 euro. The largest non-tax revenues were in Trakai district, Neringa and Palanga town municipalities (229 euro, 201 euro and 126 euro per capita). The largest grants received Birštonas and Neringa municipalities. According to the Association of Local Authorities in Lithuania (2016), since 2016 Lithuania has only four municipalities-donors: Vilnius, Kaunas, Klaipéda and Neringa cities. We have to mention that, municipal-donor means, if the municipality's income tax per capita is higher than the national average. 
Table 5. Lithuanian municipalities' ranking by the budget expenditure per capita (2005-2014).

Source: Own research.

\begin{tabular}{|c|c|c|c|c|c|c|c|c|c|c|c|c|c|c|}
\hline Municipality & $\begin{array}{c}\text { Average } \\
\text { budget } \\
\text { expenditure } \\
\text { per capita, } \\
\text { thousand } \\
\text { EUR }\end{array}$ & Rank & Municipality & $\begin{array}{c}\text { Average } \\
\text { budget } \\
\text { expenditure } \\
\text { per capita, } \\
\text { thousand } \\
\text { EUR }\end{array}$ & Rank & Municipality & $\begin{array}{c}\text { Average } \\
\text { budget } \\
\text { expenditure } \\
\text { per capita, } \\
\text { thousand } \\
\text { EUR }\end{array}$ & Rank & Municipality & $\begin{array}{c}\text { Average } \\
\text { budget } \\
\text { expenditure } \\
\text { per capita, } \\
\text { thousand } \\
\text { EUR }\end{array}$ & Rank & Municipality & $\begin{array}{c}\text { Average } \\
\text { budget } \\
\text { expenditure } \\
\text { per capita, } \\
\text { thousand } \\
\text { EUR }\end{array}$ & Rank \\
\hline Neringa & 2,910 & 1 & $\begin{array}{l}\text { Kelme } \\
\text { district }\end{array}$ & 0,693 & 13 & $\begin{array}{l}\text { Sakiai } \\
\text { district }\end{array}$ & 0,657 & 25 & Kalvarija & 0,627 & 37 & $\begin{array}{l}\text { Alytus } \\
\text { district }\end{array}$ & 0,592 & 48 \\
\hline Birštonas & 1,449 & 2 & $\begin{array}{l}\text { Skuodas } \\
\text { district }\end{array}$ & 0,685 & 14 & $\begin{array}{c}\text { Radviliskis } \\
\text { district }\end{array}$ & 0,654 & 26 & $\begin{array}{l}\text { Prienai } \\
\text { district }\end{array}$ & 0,624 & 38 & Marijampole & 0,587 & 49 \\
\hline Palanga & 1,039 & 3 & $\begin{array}{l}\text { Lazdijai } \\
\text { district }\end{array}$ & 0,681 & 15 & $\begin{array}{c}\text { Elektrènai } \\
\text { district }\end{array}$ & 0,653 & 27 & $\begin{array}{c}\text { Kaunas } \\
\text { city }\end{array}$ & 0,624 & 39 & $\begin{array}{l}\text { Siauliai } \\
\text { district }\end{array}$ & 0,584 & 50 \\
\hline $\begin{array}{l}\text { Akmené } \\
\text { district }\end{array}$ & 0,791 & 4 & Rietavas & 0,680 & 16 & $\begin{array}{l}\text { Kedainiai } \\
\text { district }\end{array}$ & 0,651 & 28 & $\begin{array}{c}\text { Vilkaviskis } \\
\text { district }\end{array}$ & 0,624 & 40 & $\begin{array}{l}\text { Kretinga } \\
\text { district }\end{array}$ & 0,578 & 51 \\
\hline Pagègiai & 0,770 & 5 & $\begin{array}{l}\text { Telšiai } \\
\text { district }\end{array}$ & 0,675 & 17 & $\begin{array}{c}\text { Mażeilkiai } \\
\text { district }\end{array}$ & 0,649 & 29 & $\begin{array}{l}\text { Siauliai } \\
\text { city }\end{array}$ & 0,622 & 41 & $\begin{array}{c}\text { Pasvalys } \\
\text { district }\end{array}$ & 0,574 & 52 \\
\hline Druskininkai & 0,751 & 6 & Visaginas & 0,674 & 18 & $\begin{array}{l}\text { Molètai } \\
\text { district }\end{array}$ & 0,649 & 30 & $\begin{array}{l}\text { Kaišia dorys } \\
\text { district }\end{array}$ & 0,622 & 42 & $\begin{array}{l}\text { Vilnius } \\
\text { city }\end{array}$ & 0,554 & 53 \\
\hline $\begin{array}{c}\text { Zarasai } \\
\text { district }\end{array}$ & 0,719 & 7 & $\begin{array}{l}\text { Anykščiai } \\
\text { district }\end{array}$ & 0,672 & 19 & $\begin{array}{l}\text { Ukmerge } \\
\text { district }\end{array}$ & 0,636 & 31 & $\begin{array}{l}\text { Salc̈ininkai } \\
\text { district }\end{array}$ & 0,619 & 43 & $\begin{array}{c}\text { Rokiskkis } \\
\text { district }\end{array}$ & 0,544 & 54 \\
\hline $\begin{array}{c}\text { Joniskis } \\
\text { district }\end{array}$ & 0,717 & 8 & $\begin{array}{c}\text { Siluté } \\
\text { district }\end{array}$ & 0,666 & 20 & $\begin{array}{l}\text { Trakai } \\
\text { district }\end{array}$ & 0,635 & 32 & $\begin{array}{l}\text { Taurage } \\
\text { district }\end{array}$ & 0,617 & 44 & $\begin{array}{l}\text { Biržai } \\
\text { district }\end{array}$ & 0,537 & 55 \\
\hline $\begin{array}{c}\begin{array}{c}\text { Silalé } \\
\text { district }\end{array} \\
\end{array}$ & 0,709 & 9 & $\begin{array}{l}\text { Jonava } \\
\text { district }\end{array}$ & 0,663 & 21 & $\begin{array}{l}\text { Utena } \\
\text { district }\end{array}$ & 0,631 & 33 & LIIHUANIA & 0,612 & & $\begin{array}{c}\text { Klaipeda } \\
\text { district }\end{array}$ & 0,536 & 56 \\
\hline $\begin{array}{c}\text { Ignalina } \\
\text { district }\end{array}$ & 0,707 & 10 & $\begin{array}{c}\text { Jurbarkas } \\
\text { district }\end{array}$ & 0,661 & 22 & $\begin{array}{l}\text { Sirvintos } \\
\text { district }\end{array}$ & 0,631 & 34 & $\begin{array}{c}\text { Klaipeda } \\
\text { city }\end{array}$ & 0,609 & 45 & $\begin{array}{l}\text { Vilnius } \\
\text { district }\end{array}$ & 0,512 & 57 \\
\hline $\begin{array}{l}\text { Varena } \\
\text { district }\end{array}$ & 0,700 & 11 & $\begin{array}{l}\text { Alytus } \\
\text { city }\end{array}$ & 0,659 & 23 & $\begin{array}{l}\text { Svencionys } \\
\text { district }\end{array}$ & 0,630 & 35 & Kazlu Ruda & 0,607 & 46 & $\begin{array}{l}\text { Kaunas } \\
\text { district }\end{array}$ & 0,502 & 58 \\
\hline $\begin{array}{l}\text { Pakruojis } \\
\text { district }\end{array}$ & 0,700 & 12 & $\begin{array}{l}\text { Raseiniai } \\
\text { district }\end{array}$ & 0,658 & 24 & $\begin{array}{l}\text { Plunge } \\
\text { district }\end{array}$ & 0,629 & 36 & $\begin{array}{c}\text { Kupiskis } \\
\text { district }\end{array}$ & 0,605 & 47 & $\begin{array}{c}\text { Paneveżys } \\
\text { district }\end{array}$ & 0,494 & 59 \\
\hline & & & & & & & & & & & & $\begin{array}{c}\text { Panevéżys } \\
\text { city }\end{array}$ & 0,488 & 60 \\
\hline
\end{tabular}


We also analyzed the municipal budget expenditures (Table 5). We emphasize that the budgetary costs are divided into general government services, defense, public order, economy, environmental protection, housing and communal economy, health care, recreation and culture, education and social protection.

We ranked municipalities in accordance with the average budget expenditure per capita and grouped them into five groups. Mid-largest budget expenditure per capita was in three municipalities: Neringa, Birštonas and Palanga (rank from 1 to 3). The second group consists of 9 municipalities (rank from 4 to 12), the third group consists of 16 municipalities (rank from 13 to 28), the fourth group is the largest - it consists of 19 municipalities (ranks from 29 to 47). The last group consists of 13 municipalities (rank from 48 to 60) where average budget expenditures are the lowest.

We noticed that, according to the functions, the greatest share of the budget expenditures (48.82 per cent) was given to education. Less money was given to another functions: social protection, general public services, and recreation and culture (14,09 per cent, 12,38 per cent and 11.18 per cent). The minimum share of expenditures was to health protection, public order and defense $(0,94$ per cent, 0,82 per cent and 0,11 per cent).

After an assessment of fiscal competitiveness in Lithuanian municipalities, we noticed that ranking and average index methods can be useful to show what the situation in fiscal competitiveness is. To sum up the results of our research, Neringa, Birštonas and Palanga city has financial autonomy in Lithuania. These municipalities collect the largest part of tax revenue compared with others, take very high rank in non-tax revenue. But, unfortunately, Neringa and Birštonas receive general grants, which show a not very good situation in fiscal competitiveness. Palanga city ranks at the position 43. What is more, the analysis showed that because of small population in these municipalities, Neringa, Birštonas and Palanga city got very good results in fiscal competitiveness. So we need to make a conclusion of ranking by surplus value per capita as well. In the ranking by surplus value per capita, the first two places are taken by Vilnius city and Klaipeda city, when Neringa is in the fifth place. That means that these three cities really are fiscally competitive.

\section{CONCLUSIONS}

The largest part of the local budget revenues consists of tax revenues and grants. Meanwhile, non-tax revenues, which the municipality has the greatest potential to influence, represent a very minor part of the total municipal income, and the value is not high. Municipalities are unable to adequately respond to economic changes. When costs increase, municipalities are faced with a constant lack of funds, because in the municipalities assigned duty officers do not perform their fiscal (accumulation) function. For this reason, 
the municipality is unable to accumulate additional funds, which are needed for unforeseen, temporary difficulties to overcome or improve the quality of service. Therefore, the municipal fiscal initiative and responsibility stay weaker and financial activities are becoming less effective.

In our research, ranking and average index methods were used to analyze and rank Lithuanian municipalities in accordance with the size of territory, population, surplus value per capita, budget revenue and expenditures. In analyzing the situation of fiscal competitiveness in Lithuanian municipalities it was noted that the most fiscally competitive municipalities are Vilnius city, Klaipeda city and Neringa. Vilnius and Klaipeda cities are two of the biggest municipalities according to population; therefore, create much more surplus value per capita than other municipalities. Neringa municipality takes a high rank in surplus value per capita as well. What is more, we ranked Neringa in one of the first positions in accordance with budget revenue and expenditures.

\section{REFERENCES}

[1] Baltušnikienė, J. (2004). Theoretical-methodological aspects of fiscal decentralization. Monetary Studies, 1, 89-102.

[2] Baltušnikienė, J., \& Astrauskas, A. (2009). Theoretical aspects of sub-national governance structure and competence establishment of territorial municipalities. Public policy and administration, 28. 7-22.

[3] Balvočiūtė, R., \& Makauskaitè, A. (2012). Regional disparities in Lithuania: local government funding. Economics and Management: Current Issues and Perspectives, 4(28). 38-52.

[4] Bessard, P. (2008). Tax competition: the Swiss case. Liberal institute, 85-104. Retrieved from http://www.libinst.ch/publikationen/Bessard-Swiss-Case.pdf.

[5] Brett, C., \& Tardif, C. (2005). Yardstick Competition and Tax Base Competition in Local Tax Setting in New Brunswick. Retrieved from http:// citeseerx.ist.psu.edu/viewdoc/download?doi=10. 1.1.498.3123\&rep=rep1\&type=pdf.

[6] Bruneckienè, J. (2010). Measurement of regional competitiveness within the country in different methods: the analysis and evaluation of results. Economics and management, 15, 25-31.

[7] Charlot, S., Paty, S., \& Piguet, V. (2010). Does fiscal cooperation increase local tax rates? Working paper, 1. 1-25.

[8] Civinskas, R., \& Tolvaišis, L. (2006). Interbudgetary Redistribution of Funds in Lithuania: Institutional Interests and Financial Independence of Local Authorities. Public policy and administration, 16. 87-99.

[9] da Silva Costa, J., \& Carvalho, A. (2013). Yardstick Competition among Portuguese Municipalities: The Case of Urban Property Tax (IMI). FEP working paper, 495. 1-24.

[10] Davulis, G. (2006). The Structure of finances and their development in Lithuanian Local government. Economics and Management: Current Issues and Perspectives, 2(7), 40-45.

[11] Davulis, G. (2009). Analysis of a situation on local taxes in Lithuania. Intellectual Economics, 1(15), 21-29.

[12] Delgado, F. J., Mayor-Fernandez, M. (2009) Tax mimicking among local governments: some evidence from Spanish municipalities. Retrieved from http://www.ub.edu/sea2009.com/ Papers/112.pdf.

[13] Domarkas, V., Laukaitytè, A., \& Mačiukas, V. (2012). Assessment of Development Level of Municipal Websites of the Republic of Lithuania. Public policy and administration, 1(11). 23-36.

[14] Gardiner, B., Martin, R., \& Tyler, P. (2004). Competitiveness, Productivity and Economic Growth across the European Regions. Retrieved from http://econpapers.repec.org/paper/wiwwiwrsa/ersa04p333.html. 
[15] Gerard, M., \& Van Malderen, L. (2012). Tax Interaction Among Walloon Municipalities: Is there Room for Yardstick Competition, Intellectual Trend and Partisan Monopoly Effect? CESifo Working Paper Series, 4025. 1-20.

[16] Ginevičius, R., Slavinskaitè, N., \& Gedvilaitė D. (2014). Regional differentiation of revenues collected by local governments of Lithuania. Business, Management and Education, 12(2). 181-193.

[17] Hawkesworth, I., Emery, R., Wehner, J., \& Saegert, J. (2010). Budgeting in Lithuania. OECD Journal on Budgeting, 3. 1-36.

[18] Hendrick, R., Wu, Y., \& Jacob, B. (2007). Tax Competition among Municipal Governments: Exit vs.Voice. Retrieved from http://localgov.fsu.edu/readings_papers/regional\%20governance/view.pdf.

[19] Janeba, E., \& Osterloh S. (2012). Tax and the City - A Theory of Local Tax Competition and Evidence for Germany. ZEW - Centre for European Economic Research Discussion Paper,12-005. $1-41$.

[20] Krumplytè, J., \& Rimkevičienè, A. (2004) The application of the rating and cluster analysis assessing the hierarchy and differences of Lithuanian regions from the social - economic point of view. Social Research, 4. 39-48.

[21] Lazauskienè, A. (2008). The search for the optimal size of municipalities. Economics and Management: Current Issues and Perspectives, 1(10), 92-98.

[22] Lithuanian statistical system (2016). Official Statistics Portal. Retrieved from http://www.stat. gov.lt/en/;jsessionid=3DB2CC22F135DE84AFF6F4C473788ECB.

[23] Lyytikainen, T. (2011). Tax Competition Among Local Governments: Evidence from a Property Tax Reform in Finland. SERC discussion paper 82. Retrieved from http://www.spatialeconomics.ac.uk/textonly/SERC/publications/download/sercdp0082.pdf.

[24] Martin, R. (2003). A study on the Factors of Regional Competitiveness. A draft final report for the European Commission Directorate - General regional policy. Retrieved from http:/ / ec.europa. eu/regional_policy/sources/docgener/studies/pdf/3cr/competitiveness.pdf.

[25] Misiunas, A., Svetikas, Z. (2003). Evaluation of economic disparities in Lithuanian regions. Economics, 64. 84-91.

[26] Monten, A., \& Thum, M. (2010). Ageing municipalities, gerontocracy and fiscal competition. European Journal of Political Economy, 26(2). 235-247.

[27] Nefas, S. (2013). Measurement of Sociality in Lithuanian Municipalities: The Case of the Biržai District Municipality. Social Research, 1(30), 96-103.

[28] Novošinskienè, A. (2013). Lithuanian tax system evaluation in the fiscal tax function aspect. Agricultural Sciences, 20(3), 222-229.

[29] Sedmihradska, L., \& Čabla, A. (2013). Budget accuracy in Czech municipalities and the determinants of tax revenue forecasting errors. Ekonomická revue - Central European Review of Economic Issues, 16. 197-206.

[30] Šeputienė, J., \& Brazauskienė, K. (2013). Estimation of Lithuania's competitiveness in the context of EU member states. Economics and Management: Current Issues and Perspectives, 1(29). 30-37.

[31] Stankevičius, E. (2014). Assessment of the tax burden impact on competitiveness of economy in context of the social environment (Doctoral dissertation). Retrieved from http://vddb.library.lt/ fedora/get/LT-eLABa-0001:E.02 2014 D_20141105_162130-57417/DS.005.0.01.ETD.

[32] The Association of Local Authorities in Lithuania. (2016). Next year in Lithuania - four municipalities-donors. Retrieved from http://www.lsa.lt/lt/ home/8-naujienos/890-kitais-metais-lietuvoje-jau-keturios-savivaldybes-donores.

[33] The Kosovo municipal competitiveness index report. (2013). Retrieved from http://www.uboconsulting.com/publications/MCI_2013_ENG.pdf.

[34] The Law on the Budget Structure. (2014). Retrieved from http://www3.lrs.lt/pls/inter3/dokpaieska.showdoc_l?p_id $=468453$.

[35] Žalevičienè, A. (2012). Regional Policy and Structural Support of the EU: Evaluation Experience. Economics and Management: Current Issues and Perspectives, 2(26), 44-53. 